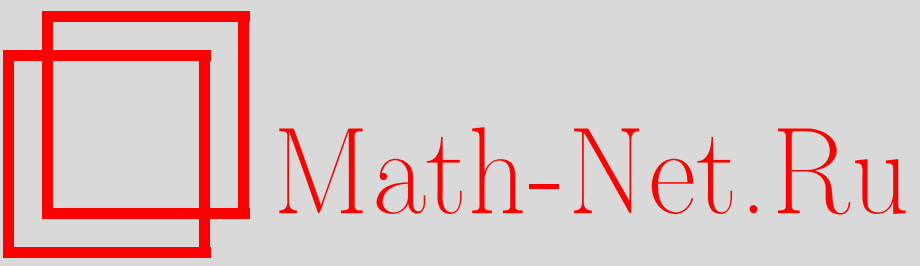

В. Г. Кадышевский, В. Н. Родионов, Поляризация электрон-позитронного вакуума сильным магнитным полем в теории с фундаментальной массой, $T M \Phi$, 2003, том 136, номер 3, 517-528

DOI: https://doi.org/10.4213/tmf1916

Использование Общероссийского математического портала Math-Net.Ru подразумевает, что вы прочитали и согласны с пользовательским соглашением

http: //www . mathnet.ru/rus/agreement

Параметры загрузки:

IP : 54.205 .225 .156

26 апреля 2023 г., 12:21:50 
ТЕОРЕТИЧЕСКАЯ

И МАТЕМАТИЧЕСКАЯ

ФИЗИКА

Том 136, № 3

сентябрь, 2003

(C) 2003 г.

В.Г. Кадышевский* , В.Н. Родионов ${ }^{\dagger}$

\title{
ПОЛЯРИЗАЦИЯ ЭЛЕКТРОН-ПОЗИТРОННОГО ВАКУУМА СИЛЬНЫМ МАГНИТНЫМ ПОЛЕМ В ТЕОРИИ С ФУНДАМЕНТАЛЬНОЙ МАССОЙ
}

\begin{abstract}
В рамках теории с фундаментальной массой в однопетлевом приближении вычислена точная лагранжева функция интенсивного постоянного магнитного поля, заменяющая собой лагранжиан Гейзенберга-Эйлера в традиционной КЭД. Установлено, что найденное обобщение лагранжевой функции вещественно при произвольных значениях магнитного поля. В слабом поле вычисленный лагранжиан совпадает с известной формулой Гейзенберга-Эйлера. При экстремально сильных полях зависимость лагранжиана от поля полностью исчезает, и в этой области он стремится к предельному значению, которое определяется отношением фундаментальной и лептонной масс.
\end{abstract}

Ключевые слова: фундаментальная масса, поляризация вакуума, сильные поля.

Хотя первые расчеты эффектов поляризации вакуума внешними интенсивными электромагнитными полями были выполнены еше в 1936 г. (лагранжиан Гейзенберга-Эйлера (ГЭ) [1]), до сих пор эти явления продолжают оставаться в центре внимания и широко изучаются в рамках ковариантной теории возмущений как радиационные поправки высших порядков с диаграммами, содержашими различное число электронных петель [2]-[5]. Проведенные оценки свидетельствуют о том, что лишш в экспоненциально сильных электромагнитных полях ${ }^{1)}$

$$
F_{\mathrm{c}} \sim e^{3 \pi / \alpha} H_{\mathrm{c}}
$$

квантовые (радиационные) поправки могли бы достичь величины максвелловской плотности энергии электромагнитного поля [6].

\footnotetext{
1) Здесь $H_{\mathrm{c}}=m^{2} /|e|=4.41 \cdot 10^{13} \Gamma \mathrm{c}$ - характерный масштаб напряженности электромагнитных полей в КЭД, $е$ и $m$ - заряд и масса электрона, $\alpha=e^{2}=1 / 137$ - постоянная тонкой структуры (использована система единиц $\hbar=c=1$ ).

* Объединенный институт ядерных исследований, Дубна, Московская обл., Россия. E-mail: kadyshev@jinr.ru

${ }^{\dagger}$ Московская государственная геологоразведочная академия, Москва, Россия. E-mail: physics@msgpa.msgpa.ru
} 
Как известно, вычисления, проведенные Гейзенбергом и Эйлером, не содержат приближений по интенсивности внешних электромагнитных полей, а полученные ими результаты многократно подтверждены расчетами в рамках иных подходов. На этом основании в ряде работ делался вывод о том, что величина (1) и является предельной напряженностью поля в стандартной квантовой электродинамике (КЭД) (см., например, [7]). В действительности вопрос о существовании столь сильных полей $\left(F_{\mathrm{c}} \sim 10^{560} H_{\mathrm{c}}\right)$ является риторическим хотя бы потому, что расстояния, на которых подобные поля могут возникать (например, вблизи атомных ядер), оказываются на много порядков меньше масштаба "планковской длины" $l_{\mathrm{P}}=\sqrt{G \hbar / c^{3}} \sim 10^{-33}$ см. Обшеизвестно, что на расстояниях $l \sim l_{\mathrm{P}}$ должны проявляться эффекты квантовой гравитации, рассмотрение которых в рамках сушествуюшей теории заведомо лишено смысла. Вместе с тем поиск воз-

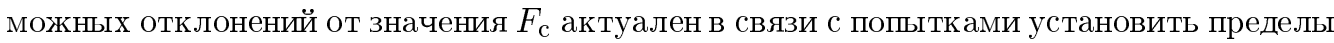
справедливости стандартной КЭД. Очевидно, что неразрешимость вопроса о границах применимости традиционной КЭД в рамках самой существующей теории диктует необходимость выхода за ее пределы.

В этой связи представляет интерес сравнить радиационные поправки к максвелловской лагранжевой функции постоянного поля, рассчитанные в рамках сушествуюшей КЭД, с результатами, которые можно получить из модифицированной теории. Одним из возможных вариантов обобщения традиционной квантовой теории поля (КТП) является теория с фундаментальной массой (см. [8]-[10] и цитируемые там работы). Отправным пунктом этой теории является условие ограниченности спектра масс элементарных частиц, представимое в виде

$$
m \leqslant M,
$$

где новый универсальный параметр $M \geqslant 1$ ТэВ носит название фундаментальной мас$c b l$. Соотношение (2) используется как некий дополнительный фундаментальный физический принцип, который и положен в основу новой КТП.

Необходимо подчеркнуть, что новая формулировка КТП, несмотря на присутствие в ней фундаментальной массы, остается локальной схемой, допускающей использование калибровочных групп симметрии. В полном лагранжиане новой теории возникает ряд дополнительных членов с константами связи, пропорциональными $M^{-1}$ и $M^{-2}$, что кардинально меняет картину взаимодействия частиц при высоких энергиях $(E \geqslant M)$. Благодаря ограничению на спектр масс (2) комптоновская длина волны частицы $\lambda_{\mathrm{C}}=$ $\hbar /(m c)$ не может быть меньше фундаментальной длины $l=\hbar /(M c)$, что вносит в теорию естественное ограничение на возможность пространственной локализации элементарных частиц.

Говоря о физике сверхмалых расстояний, нельзя не сказать о глубокой аналогии ${ }^{2)}$, сушествуюшей между явлениями, возникаюшими при больших переданных импульсах, и процессами, идушими в интенсивных электромагнитных полях [2]-[4], [11]-[16]. Пересечение, казалось бы, совершенно различных областей физики на самом деле не случайно и подсказывается простыми соображениями размерности. Действительно, в принятой системе единиц $(\hbar=c=1)$ электрический заряд $e=1 / \sqrt{137}$ безразмерен, а импульс и вектор-потенциал электромагнитного поля имеют одинаковую размерность об-

\footnotetext{
${ }^{2)}$ На эту аналогию впервые обратили внимание Мигдал [11] и Ритус [2].
} 
ратной длины $l^{-1}$. Размерность напряженности поля есть $l^{-2}$, т.е. совпадает с размерностью квадрата энергии или массы. Таким образом, когда интенсивность электромагнитного поля сравнивается с квадратом массы частицы, это значение (с точностью до константы) определяет некоторое характерное для нее поле. В частности, легко установить, что электрическое поле $\mathcal{E}=m^{2} / e$ на комптоновской длине волны $\lambda_{\mathrm{C}}=1 / m$ производит работу, равную энергии покоя частицы. Энергия врашения заряженной частицы в магнитном поле с интенсивностью $H=m^{2} / e$ также совпадает с $m$, а радиус орбиты для низшего уровня Ландау $r_{H}$ сравнивается с $\lambda_{\mathrm{C}}{ }^{3)}$.

Дальнейшее повышение интенсивности поля $F>m^{2} / e$ еше больше сокрашает пространственные границы формирования процессов. Таким образом, размеры области взаимодействия, определяемые величиной поля в пределе $F \gg m^{2} / e$, могут иметь весьма малые масштабы. Например, в случае электрона для магнитных полей $H \gg H_{\mathrm{c}}=$ $m^{2} / e=4.41 \cdot 10^{13}$ Гс получаем

$$
r_{H} \approx \lambda_{\mathrm{C}}\left(\frac{H_{\mathrm{c}}}{H}\right)^{1 / 2} \ll \lambda_{\mathrm{C}} .
$$

Важным аспектом рассматриваемой проблемы является то, что современный уровень развития лазерной физики [17] позволяет приступить к постановке ряда оптических экспериментов для непосредственного измерения величин вкладов, обусловленных нелинейными вакуумными эффектами, предсказываемыми различными обобщениями электродинамики Максвелла [18]. В этой связи следует подчеркнуть, что экспериментальная проверка нелинейных эффектов в вакууме, проводимая с высокой точностью в присутствии сравнительно слабых электромагнитных полей, может дать также ценную информацию и о правильности предсказаний КЭД на малых расстояниях [15], [16]. Отметим попутно, что в том же смысле актуальны прецизионные измерения различных величин (например, аномальных магнитных моментов (АММ) электрона и мюона), проводимые в нерелятивистской области энергий, наряду с изучением взаимодействия частиц при высоких энергиях.

Серьезного внимания заслуживает и то, что вычисления лагранжиана, заменяющего собой лагранжиан ГЭ, можно провести, сохранив метод точных решений уравнения Дирака в электромагнитных полях произвольной интенсивности. В этом подходе предлагаемое теоретическое обобщение, так же как и традиционная КЭД, изначально не содержит ограничений на интенсивности электромагнитных полей.

В настоящей работе в рамках теории с фундаментальной массой мы рассмотрим поправку к лагранжевой функции электромагнитного поля, обусловленную поляризацией вакуума в присутствии сколь угодно сильного постоянного магнитного поля. Для решения этой задачи, так же как и в известном подходе [6], удобно представить электрон-позитронный вакуум как систему электронов, заполняющих уровни с "отрицательной” энергией. Существенным отличием от традиционных расчетов является то, что

\footnotetext{
${ }^{3)}$ Отметим, что за величиной $r_{H}$ закрепилось специальное название - магнитная длина, широко используемое в физике полупроводников.
} 
в КЭД с фундаментальной массой заряженные лептоны обладают магнитными моментами, не равными магнетону Бора. В новом лагранжиане электромагнитного взаимодействия помимо традиционного "минимального" члена $e \bar{\Psi} \gamma_{\mu} \Psi A^{\mu}$ присутствуют "неминимальные" слагаемые, в частности

$$
-\frac{e}{2 m}\left(\sqrt{1+\frac{m^{2}}{M^{2}}}-1\right) \bar{\Psi} \sigma^{\mu \nu} \Psi F_{\mu \nu}
$$

где $F_{\mu \nu}$ - тензор электромагнитного поля.

Таким образом, для частицы с массой $m$ имеем

$$
\mu=\mu_{0} \sqrt{1+\frac{m^{2}}{M^{2}}}
$$

где $\mu_{0}=e /(2 m)$ - магнетон Бора, $M$ - фундаментальная масса. Тем самым в модифицированной КЭД электрон изначально обладает АММ, которьй равен

$$
\Delta \mu=\mu-\mu_{0}=\mu_{0}\left(\sqrt{1+\frac{m^{2}}{M^{2}}}-1\right)
$$

Заметим, что для постоянного и однородного магнитного поля уравнение Дирака-Паули

$$
\left(\widehat{P}-m-\frac{\Delta \mu}{2} \sigma_{\mu \nu} F^{\mu \nu}\right) \psi(x)=0
$$

имеет точное решение [19]. При этом собственные значения оператора Гамильтона уравнения (7) явным образом зависят от ориентации спина по отношению к оси симметрии, задаваемой направлением магнитного поля. Таким образом, спектр энергий электрона, движушегося в постоянном однородном магнитном поле произвольной интенсивности, имеет вид

$$
E_{n}(p, H, \xi)=m \sqrt{\frac{p^{2}}{m^{2}}+\left(\sqrt{\left|\frac{H}{H_{\mathrm{c}}}\right|(1+2 n+\xi)+1}+\xi \frac{\mu-\mu_{0}}{2 \mu_{0}} \frac{H}{H_{\mathrm{c}}}\right)^{2}}
$$

где $p$ - проекция импульса электрона на направление внешнего поля $H, n=0,1,2, \ldots-$ квантовое число, нумерующее уровни Ландау, величина $\xi= \pm 1$ характеризует проекцию спина электрона на направление магнитного поля. В основном состоянии энергию электрона можно записать как

$$
E_{0}(0, H,-1)=m\left|1-\frac{H}{2 H_{\mathrm{c}}} \frac{\mu-\mu_{0}}{\mu_{0}}\right| .
$$

Из (6) следует, что в пределе $M \gg m$ относительная величина АММ равна

$$
\frac{\Delta \mu}{\mu_{0}}=\frac{m^{2}}{2 M^{2}} .
$$


Полагая в $(9) H=4(M / m)^{2} H_{\mathrm{c}}=4 H_{\mathrm{c}}^{*}$, мы получаем $E_{0}=0$, т.е. при таких значениях магнитного поля отсутствует энергетическая шель между электронными и позитронными состояниями. Однако эта неожиданная и далеко не тривиальная особенность энергетического спектра вакуумных электронов, возникающая в теории с фундаментальной массой, при дальнейшем росте поля исчезает. Действительно, из (9) непосредственно видно, что при $H>4 H_{\text {c }}^{*}$ ширина шели с увеличением $H$ вновь растет. Следует также заметить, что “схлопьвание” энергетической шели происходит, когда удвоенное значение магнитной длины $r_{H}$ (диаметр орбиты электрона) сравнивается с фундаментальной длиной $l$. В рамках традиционной КЭД подобные эффекты не возникают, что обусловлено динамической природой АММ и его монотонным убыванием от швингеровского значения $\alpha /(2 \pi)$ до нуля с ростом интенсивности поля (см., например, [12], [14]).

Учитывая нетривиальную специфику поведения энергии вакуумного электрона в теории с фундаментальной массой, в рамках однопетлевого приближения получим точное выражение для функции Лагранжа в сильном постоянном магнитном поле. Замечая, что радиационная добавка к классической плотности функции Лагранжа совпадает с точностью до знака с полной плотностью энергии электрон-позитронного вакуума при наличии внешнего поля [6],

$$
\mathcal{L}^{\prime}=-W^{H}
$$

рассчитаем $W^{H}$ в постоянном магнитном поле с учетом АММ электрона. Не останавливаясь на деталях стандартных расчетов, представим $W^{H}$ в виде

$$
W^{H}=-\frac{|e H|}{(2 \pi)^{2}} \int_{-\infty}^{\infty} d p\left[-\varepsilon_{0}^{+}(p)+\sum_{n=0}^{\infty}\left[\varepsilon_{n}^{-}(p)+\varepsilon_{n}^{+}(p)\right]\right]
$$

где

$$
\varepsilon_{n}^{ \pm}=\sqrt{p^{2}+m^{2}\left(\sqrt{1+2 \frac{H}{H_{\mathrm{c}}}} n \pm \frac{H}{4 H_{\mathrm{c}}^{*}}\right)^{2}} .
$$

Используя интегральные преобразования Лапласа и Фурье для функций, определяющих (11), и проводя суммирование по уровням Ландау, для $\mathcal{L}^{\prime}$ можно получить

$$
\begin{aligned}
\mathcal{L}^{\prime}= & -\frac{m^{4} \gamma b_{1}}{8 \pi^{2}} \int_{0}^{\infty} \frac{d \eta}{\eta^{2}} e^{-\eta}[\operatorname{sh} b+ \\
& \left.+\frac{1}{\pi} \int_{-\infty}^{\infty} \frac{e^{-i x} d x}{x} \operatorname{ctg}\left(-\frac{i \gamma \eta}{b_{1}}+x \gamma\right){ }_{1} F_{2}\left(\{1\},\left\{\frac{1}{4}, \frac{3}{4}\right\},-\frac{b^{4}}{64 x^{2}}\right)\right],
\end{aligned}
$$

где введены обозначения $b_{1}=1+\left(H /\left(4 H_{\mathrm{c}}^{*}\right)\right)^{2}, b=a / b_{1}, a=\eta H /\left(2 H_{\mathrm{c}}^{*}\right), \gamma=H / H_{\mathrm{c}}$, $H_{\mathrm{c}}^{*}=M^{2} / e, \mathrm{a}_{1} F_{2}(z)$ - обобщенная гипергеометрическая функция. Формула (13) представляет собой точное выражение для функции Лагранжа в модифицированной КЭД, рассчитанное в однопетлевом приближении в магнитном поле произвольной интенсивности. Важное отличие от лагранжиана ГЭ состоит в том, что формула (13) содержит дополнительный полевой масштаб

$$
H_{\mathrm{c}}^{*}=\frac{M^{2}}{e}=\frac{M^{2}}{m^{2}} H_{\mathrm{c}}
$$


который естественно назвать фундаментальным полем.

Перейдя в (13) к пределам интегрирования по $x$ от 0 до $\infty$ и воспользовавшись свойством четности функции ${ }_{1} F_{2}(z)$, имеем

$$
\begin{aligned}
\mathcal{L}^{\prime}= & -\frac{m^{4} \gamma b_{1}}{8 \pi^{2}} \int_{0}^{\infty} \frac{d \eta}{\eta^{2}} e^{-\eta} \times \\
& \times\left[\operatorname{sh} b+\frac{2}{\pi} \int_{0}^{\infty} \frac{d x}{x} \frac{\sin 2 \gamma x \cos x+\sin x \operatorname{sh} y}{\operatorname{ch} 2 y-\cos 2 \gamma x}{ }_{1} F_{2}(z)\right],
\end{aligned}
$$

где $y=\eta \gamma / b_{1}, z=-b^{4} /\left(64 x^{2}\right)$. Из (15), в частности, следует, что $\operatorname{Im} \mathcal{L}^{\prime}=0$.

Вещественность лагранжиана $\mathcal{L}^{\prime}$ при всех возможных значениях поля указьвает на отсутствие нестабильных мод, т.е. вакуум в постоянном и однородном магнитном поле в теории с фундаментальной массой, так же как и в традиционной КЭД, устойчив по отношению к спонтанному образованию электрон-позитронных пар.

Выделим далее в выражении (15) интеграл по $x$. После ряда очевидных замен он приводится к виду

$$
I=\int_{0}^{\infty} \frac{d u\left[2 a_{2} \sin u \cos b_{2} u+\left(1-a_{2}^{2}\right) \sin b_{2} u\right]}{u\left[1+a_{2}^{2}-2 a_{2} \cos u\right]}{ }_{1} F_{2}\left(z_{1}\right),
$$

где $a_{2}=e^{-2 y}, b_{2}=(2 \gamma)^{-1}, z_{1}=-b^{4}(2 \gamma)^{2} /\left(64 u^{2}\right)$. Учитывая справедливость разложений

$$
\begin{aligned}
& \frac{\sin u}{1+a_{2}^{2}-2 a_{2} \cos u}=\sin u+a_{2} \sin 2 u+a_{2}^{2} \sin 3 u+\cdots \\
& \frac{1-a_{2}^{2}}{1+a_{2}^{2}-2 a_{2} \cos u}=1+2 a_{2} \cos u+2 a_{2}^{2} \cos 2 u+\cdots
\end{aligned}
$$

для интеграла (16) получим

$$
I=\int_{0}^{\infty} \frac{d u}{u}\left[-\sin b_{2} u+2 \sum_{k=0}^{\infty} a_{2}^{k} \sin \left[u\left(k+b_{2}\right)\right]\right]{ }_{1} F_{2}\left(z_{1}\right) .
$$

Легко видеть, что в поле, которое является слабым по сравнению с фундаментальным полем $H_{\mathrm{c}}^{*}$, мы можем воспользоваться разложением функции ${ }_{1} F_{2}\left(z_{1}\right)$ в нуле

$$
{ }_{1} F_{2}\left(z_{1}\right)=1+\frac{16}{3} z_{1}+\frac{256}{105} z_{1}^{2}+\cdots
$$

и для (17) получить

$$
I=\frac{\pi}{2} \frac{1+a_{2}}{1-a_{2}}=\frac{\pi}{2} \operatorname{cth} y
$$

где $y=\eta \gamma$.

Подставляя (19) в (15) и проводя стандартную регуляризацию полученного интеграла [6], имеем

$$
\mathcal{L}^{\prime}=-\frac{m^{4}}{8 \pi^{2}} \int_{0}^{\infty} \frac{e^{-\eta}}{\eta^{3}}\left[\eta \gamma \operatorname{cth} \eta \gamma-1-\frac{\eta^{2} \gamma^{2}}{3}\right] d \eta .
$$


Таким образом, из (20) следует, что в пределе "слабого" поля формула (15) совпадает с результатом ГЭ [1] для случая постоянного однородного магнитного поля произвольной интенсивности.

Рассмотрим далее область $H>4 H_{\mathrm{c}}^{*}$. Нетрудно убедиться, что в пределе сверхсиль-

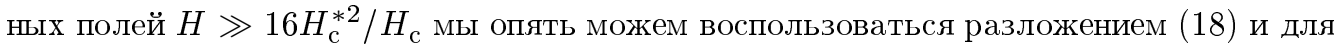
интеграла (17) получить выражение

$$
I=\frac{\pi}{2} \operatorname{cth} y,
$$

где $y=16 \eta H_{\mathrm{c}}^{* 2} /\left(H_{\mathrm{c}} H\right)$. Результаты (19) и (21) имеют простой смысл: при достаточно широкой энергетической щели, разделяющей электронные и позитронные состояния (см. формулы (8) и (9)), основной вклад в интеграл (17) формируют слагаемые с большими номерами $k$. Однако в области магнитных полей, близких к значению фундаментального поля $H \sim 4 H_{\mathrm{c}}^{*}$, т.е. когда ширина шели близка к нулю, в подынтегральной сумме (17) основной вклад дает слагаемое с $k=0$. Интеграл (17) в этом случае может быть вычислен точно. Расчеты приводят к простому результату:

$$
I=\frac{\pi}{2} \operatorname{ch}\left[\eta \frac{H}{2 H_{\mathrm{c}}^{*}} \frac{1}{1+\left(H /\left(4 H_{\mathrm{c}}^{*}\right)\right)^{2}}\right] .
$$

Нетрудно убедиться, что оценки интеграла (17), полученные в трех областях изменения магнитного поля, можно объединить и записать в виде единой асимптотической формулы

$$
I=\frac{\pi}{2} \operatorname{ch}\left[\eta \frac{H}{2 H_{\mathrm{c}}^{*}} \frac{1}{1+\left(H /\left(4 H_{\mathrm{c}}^{*}\right)\right)^{2}}\right] \operatorname{cth}\left[\frac{\eta \gamma}{1+\left(H /\left(4 H_{\mathrm{c}}^{*}\right)\right)^{2}}\right] .
$$

Подставляя (23) в (15) и проводя регуляризацию оставшегося расходяшегося интеграла ${ }^{4)}$, для $\mathcal{L}^{\prime}$ получим

$$
\mathcal{L}^{\prime}=-\frac{m^{4}}{8 \pi^{2}} \int_{0}^{\infty} \frac{d \eta}{\eta^{3}} e^{-\eta}\left[\eta b_{1} \gamma \operatorname{cth}\left(\eta \frac{\gamma}{b_{1}}\right)-b_{1}^{2}-\frac{\eta^{2}}{3} \gamma^{2}\right] .
$$

В слабых полях $H \ll H_{\text {c }}$, а также в пределе очень сильных магнитных полей $H \gg$ $4 H_{\mathrm{c}}^{*}$ подынтегральное выражение (24) можно разложить в ряд и в первом приближении получить

$$
\mathcal{L}^{\prime}=\frac{m^{4} \gamma^{4}}{360 \pi^{2} b_{1}^{2}}
$$

Таким образом, квантовая добавка к максвелловской лагранжевой функции в пределе

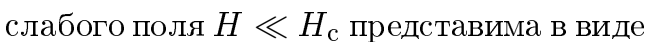

$$
\mathcal{L}^{\prime}=\frac{m^{4}}{360 \pi^{2}} \frac{H^{4}}{H_{\mathrm{c}}^{4}}+O\left[\frac{H^{6}}{H_{\mathrm{c}}^{6}}, \frac{H^{6}}{H_{\mathrm{c}}^{4} H_{\mathrm{c}}^{* 2}}\right],
$$

\footnotetext{
4) Отметим, что, во-первых, как обычно [6], следует отбросить часть интеграла, не содержащую напряженность магнитного поля и представляющую собой энергию свободных вакуумных электронов. Во-вторых, необходимо вычесть вклад, пропорциональный $H^{2}$, который уже включен в невозмущенную энергию поля. Отбрасывание этого слагаемого связано с перенормировкой напряженности поля, а тем самым и заряда. Вычитание вклада порядка $H^{4} / H^{* 4}$, по существу, соответствует перенормировке дополнительного параметра теории - фундаментальной массы и как следствие AMM частицы.
} 
где первый член совпадает с известной формулой ГЭ.

В сильном поле $H \gg 4 H_{\mathrm{c}}^{*}$ из $(25)$ можно получить

$$
\mathcal{L}^{\prime}=\frac{256 m^{4}}{360 \pi^{2}} \frac{H_{\mathrm{c}}^{* 4}}{H_{\mathrm{c}}^{4}}\left(1-\frac{32 H_{\mathrm{c}}^{* 2}}{H^{2}}\right) .
$$

Согласно (27) в пределе экстремально сильных полей $\mathcal{L}^{\prime}$ перестает зависеть от поля, т.е. квантовая поправка к плотности функции Лагранжа в теории с фундаментальной массой с ростом поля асимптотически стремится к константе

$$
\mathcal{L}_{\infty}^{\prime}=\frac{256 M^{8}}{360 \pi^{2} m^{4}}
$$

Полученный результат в каком-то смысле можно сопоставить с ситуацией, наблюдающейся в Стандартной модели $(\mathrm{CM})$, когда для ряда процессов рост сечений с ростом энергии останавливается, если в рассмотрение наряду с фотоном, векторными $W^{ \pm}$и $Z^{0}$-бозонами включается дополнительная диаграмма с хиггсовским $H$-бозоном. Учет этой диаграммы сокращает растушие члены в амплитуде и приводит к поведению сечений, согласующемуся с унитарным пределом. Как известно, СМ не предсказывает массу $H$-бозона, поэтому не исключено, что данная частица значительно тяжелее $t$-кварка, самой тяжелой из известных ныне элементарных частиц. Таким образом, может оказаться, что $M_{H} \sim 1$ ТэВ и является предельной массой, ограничивающей спектр масс элементарных частиц, т.е. играет роль фундаментальной массы (см. (2) $)^{5)}$.

Сопоставляя поправку $\mathcal{L}^{\prime}$ с лагранжевой функцией максвелловского поля, найдем интенсивность поля

$$
F_{\mathrm{c}}^{*}=\sqrt{\frac{256 \alpha}{45 \pi}} \frac{H_{\mathrm{c}}^{* 2}}{H_{\mathrm{c}}}
$$

при которой они сравниваются.

Таким образом, мы получили ясный ответ на сформулированный в начале статьи вопрос о границах применимости обобщенной КЭД, содержащей новый универсальный параметр - фундаментальную массу. Результатом появления нового полевого масштаба является и то, что в сильном поле $H>F_{\text {с }}^{*}$ квантовые поправки $\mathcal{L}^{\prime}$ вновь оказьваются малыми по сравнению с лагранжевой функцией классического максвелловского поля.

Полученные результаты могут представить интерес в связи с построением астрофизических моделей, в частности при изучении экстремально намагниченных нейтронных звезд - "магнетаров", интерес к сушествованию которых заметно возрос в последнее время (см., например, [5], [20]). Согласно моделям макроскопической намагниченности тел, состоящих в основном из нейтронов, индукция "вмороженных" в них магнитных полей увеличивается от поверхности к центральным областям и может достигать $10^{15}-10^{17}$ Гс [21].

Подчеркнем, что усиление радиационных эффектов посредством внешних интенсивных электромагнитных полей может иметь место не только для абелевых, но и в случае

\footnotetext{
5) Заметим в этой связи, что центральным пунктом программы исследований на Большом адронном калориметре (LHC) в ЦЕРН является поиск хиггсовских бозонов в диапазоне значений масс до 1 ТэВ.
} 
неабелевых КТП. В этой связи отметим, что к нетривиальным результатам приводит, например, учет в рамках СМ влияния внешнего поля на такие характеристики, как масса и магнитный момент лептонов [22], [23].

Однопетлевой массовый оператор заряженного лептона наряду с электродинамическим содержит вклады, обусловленные взаимодействием с вакуумом $W^{ \pm}, Z^{0}$ и $H$-бозонов. В отсутствие внешнего поля вклад в радиационный сдвиг массы лептона $m$ от слабых взаимодействий мал $\left(\sim\left(m / M_{i}\right)^{2}\right.$, где $\left.i=W, Z, H\right)$ по сравнению с электромагнитным вкладом. Однако в силу динамического происхождения квантовых поправок к массам лептонов они являются сложными функциями энергии и напряженности внешних полей. Вследствие этого в ультрарелятивистском пределе в интенсивных внешних полях вклады слабых токов могут оказаться доминирующими ${ }^{6)}$.

АММ заряженных лептонов в СМ, в полной аналогии с квантовыми поправками к массам частищ, обусловлены вакуумными радиационными эффектами электромагнитных и слабых взаимодействий, а также содержат вклад адронной поляризации вакуума (АПВ). Например, для АММ мюона имеем

$$
a_{\mu}(\mathrm{CM})=a_{\mu}(\mathrm{K} Э \text { Д })+a_{\mu}(\text { слаб. })+a_{\mu}(\text { адрон. }) .
$$

Согласно теоретическим оценкам, проведенным в рамках СМ недавно [24], вклады от электромагнитных и слабых взаимодействий можно записать в виде

$$
\begin{gathered}
a_{\mu}(\mathrm{K} Э \text { Д })=11658470.57(0.29) \cdot 10^{-10}(0.25 \mathrm{ppm}), \\
a_{\mu}(\text { слаб. })=15.1(0.4) \cdot 10^{-10}(0.03 \mathrm{ppm}) .
\end{gathered}
$$

Хотя расчеты вкладов в $a_{\mu}(\mathrm{CM})$, обусловленных АПВ, имеют почти сорокалетнюю историю, на сегодняшний день величина $a_{\mu}$ (адрон.) известна с наибольшей неопределенностью (см., например, [25]-[30]). Одна из наиболее надежных оценок вкладов АПВ низшего порядка, обобшающая данные по адронному $\tau$-распаду и $e^{+} e^{-}$-аннигиляции, выглядит как [25], [26] $]^{7)}$

$$
a_{\mu}(\text { адрон. })=692(6) \cdot 10^{-10}(0.6 \mathrm{ppm})
$$

и теоретическое значение АММ мюона в СМ приобретает вид [30]

$$
a_{\mu}(\mathrm{CM})=11659177(7) \cdot 10^{-10}(0.6 \mathrm{ppm})
$$

Результаты одного из последних $(g-2)$-экспериментов по измерению АММ положительных поляризованных мюонов, проведенного на накопительном кольце со сверхпроводящими магнитами Брукхейвенской национальной лаборатории (BNL) [31], представимы в виде

$$
a_{\mu}(\text { экспер. })=11659204(7)(5) \cdot 10^{-10}(0.7 \mathrm{ppm}) .
$$

\footnotetext{
6) На эту возможность впервые было указано в работе [22].

7) $\mathrm{Cм.,} \mathrm{однако,} \mathrm{[27],} \mathrm{где} \mathrm{проведено} \mathrm{вычисление} \mathrm{вклада} \mathrm{АПВ} \mathrm{высших} \mathrm{порядков,} \mathrm{а} \mathrm{также} \mathrm{недавние}$ работы [28], [29], содержащие учет вклада диаграмм третьего порядка в $a_{\mu}$ (адрон.), обусловленного фотон-фотонным рассеянием.
} 
Полученные данные позволяют определить разность

$$
\Delta_{\mu}=a_{\mu} \text { (экспер.) }-a_{\mu}(\mathrm{CM})=27 \cdot 10^{-10},
$$

превышаюшую суммарные погрешности измерений и неопределенности теоретических оценок. Согласно последним сообщениям мюонной $(g-2)$-коллаборации BNL [31] относительная величина этого превышения составляет 2.6. Ожидается, что в ближайшем будушем будет достигнуто двукратное повышение указанной точности. Очевидно, что решение мюонной $(g-2)$-проблемы может привести к появлению новой физики, возникающей за пределами СМ.

В этой связи напомним, что АММ мюона в модифицированной теории (см. (6)) изначально содержит вклад, обусловленный наличием нового универсального параметра $M$. В соответствии с (10) имеем

$$
a_{\mu}(M)=\frac{m_{\mu}^{2}}{2 M^{2}}
$$

где $m_{\mu}$ - масса мюона. Легко видеть, что по порядку величины $a_{\mu}(M)$ совпадает с (30) при $M \sim 1$ ТэВ.

Главный вывод из сравнения приведенных оценок состоит в том, что нельзя исключить возможность совпадения наблюдаемой разности теоретических и экспериментальных результатов $\Delta_{\mu}$ с величиной $a_{\mu}(M)$. Как уже отмечалось, параметр $M$ новой теории можно связать с массой хиггсовского бозона $M_{H}$. В этом случае наличие разности между $a_{\mu}$ (экспер.) и $a_{\mu}(\mathrm{CM})$ позволяет получить весьма ценную информацию о частище, масса которой не определена в СМ. Подставляя значение $m_{\mu}$ и данные по АММ мюона в (31), нетрудно установить следующие ограничения на массу $H$-бозона:

$$
1.2 \mathrm{~T} \ni \mathrm{B} \leqslant M_{H} \leqslant 1.8 \mathrm{~T} \text { В } .
$$

Как известно, вариант СМ, в котором масса хиггсовских бозонов $M_{H} \geqslant 1$ ТэВ, влечет за собой ряд дополнительных особенностей и, в частности, невозможность описания слабых взаимодействий в секторе $H$-, $W$ - и $Z$-частиц в рамках теории возмушений [32]. Тем самым естественно возникает необходимость построения новой непертурбативной теории. Хиггсовский механизм возникновения масс и компенсации расходимостей наряду с условием ограниченности спектра масс $m \leqslant M_{H}$ (см. (2)) могут стать неотъемлемыми элементами одного из перспективных вариантов модифицированной теории "СМ с фундаментальной массой".

Еше одна весьма интересная особенность неабелевых КТП со спонтанно нарушенной симметрией обнаруживается при изучении вакуума электрослабых взаимодействий в интенсивных внешних полях (см., например, обзор [33]). Симметрия и свойства вакуума, представляющего собой конденсат нейтрального скалярного поля, в этих теориях не являются навсегда заданными и могут меняться при фазовых переходах, обусловленных, например, воздействием внешних электромагнитных полей. В частности, возникает возможность восстановления спонтанно нарушенной симметрии с помощью интенсивного магнитного поля. Нетривиальность исчезновения конденсата скаляров в магнитном поле определяется тем, что плотность энергии вакуумного состояния содержит мнимую часть, связанную с вероятностью распада вакуума. Подобная вакуумная 
нестабильность обусловлена наличием в магнитном поле состояния с "отрицательным квадратом энергии", которое интерпретируется как появление тахионной моды.

Механизм, приводящий к восстановлению спонтанно нарушенной симметрии в калибровочных теориях, аналогичен рассмотренному выше и заключается в воздействии магнитного поля $H$ на спектр вакуумных осцилляторов заряженных векторных полей. Учет значения массы векторных бозонов делает очевидным условие, согласно которому характерная величина поля должна быть экстремально большой $\left(H \sim M_{W}^{2} / e\right)$. Таким образом, анализ эффектов внешних полей в этом случае, так же как и в рассмотренном нами явлении поляризации электрон-позитронного вакуума в теории с фундаментальной массой, требует непертурбативного подхода и использования точных по полю решений [34]

$$
p_{0}^{2}=p^{2}+M_{W}^{2}+(2 n+1-2 \sigma) e H,
$$

где $M_{W}$ - масса векторного бозона, $p$ - проекция импульса вдоль поля $H, \sigma$ - спиновая переменная, принимающая значения $0, \pm 1, n=0,1,2, \ldots$ Основное состояние ( $p=0$, $n=0, \sigma=1)$

$$
p_{0}^{2}=M_{W}^{2}-e H
$$

становится тахионным при $H>M_{W}^{2} / e$. Легко видеть, что именно оно и является источником возникновения мнимой части эффективного потенциала.

Как уже отмечалось, энергия основного состояния вакуумных электронов (9) неотрицательна при любых значениях магнитного поля, и, таким образом, данный вариант КЭД свободен от тахионных мод. В случае электрического поля электрон-позитронный вакуум рассматриваемой теории, так же как и в обычной КЭД, не является стабильным, что находит отражение в отличной от нуля вероятности рождения полем $e^{+} e^{-}$-пар из вакуума. Отметим также, что учет наряду с магнитным полем действия интенсивного электрического поля может быть проведен по аналогии со случаем традиционной КЭД [6]. Подобным же образом решаются и вопросы расчета эффективного лагранжиана для скалярной КЭД, вид которого легко восстанавливается, если известен результат для электронов (см. [35]).

Благодарности. Авторы благодарны В.Р. Халилову за внимание к работе и ценные замечания. Работа выполнена при поддержке РФФИ (грант № 02-02-16784).

\section{Список литературы}

[1] W. Heisenberg, H. Euler. Z. Phys. 1936. V. 98. P. 714.

[2] В. И. Ритус. ЖЭТФ. 1975. Т. 69. С. 1517.

[3] В. И. Рumyc. ЖЭТФ. 1977. Т. 73. С. 807.

[4] V. I. Ritus. Effective Lagrange function of intense electromagnetic field in QED. In: Proc. Conf. "Frontier Test of QED and Physics of the Vacuum" (Sandanski, Bulgaria, 9-15 June, 1998). Eds. E. Zavattini, D. Bakalov, C. Rizzo. Sofia: Heron Press, 1998; hep-th/9812124.

[5] В.Р. Халилов. ТМФ. 2002. Т. 133. № 1. С. 103.

[6] В. Б. Берестецкий, Е. М. Лифииц, Л. П. Питаевский. Квантовая электродинамика. М.: Наука, 1989.

[7] M. Greenman, F. Rohrlich. Phys. Rev. D. 1973. V. 8. P. 1103.

[8] V. G. Kadyshevsky. Nucl. Phys. B. 1978. V. 141. P. 477; FERMILAB-Pub. 78-070-THY. Sept. 1978. Batavia, IL: FERMILAB, 1978; В. Г. Кадьиевский. ЭЧАЯ. 1980. Т. 11. № 1. C. 5 . 
[9] V.G. Kadyshevsky, M.D. Mateev. Phys. Lett. B. 1981. V. 106. P. 139; Nuovo Cimento A. 1985. V. 87. P. 350.

[10] В. Г. Кадышевский. ЭЧАЯ. 1998. Т. 29. № 3. С. 563.

[11] А. Б. Мигдал. ЖЭЭТ. 1972. Т. 62. С. 1621.

[12] И. М. Тернов, В.Р. Халилов, В. Н. Родионов. Взаимодействие заряженных частиц с сильным электромагнитным полем. М.: Изд-во МГУ, 1982.

[13] А.А. Гриб, С. Г. Мамаев, В. М. Мостепаненко. Квантовые эффекты в интенсивных внешних полях. М.: Атомиздат, 1980.

[14] В. И. Рumyc. Тр. ФИАН. 1979. Т. 111. С. 5.

[15] В.Г. Кадишевский, В.Н. Родионов. Электромагнитная длина и фундаментальная масса в электродинамике. В сб.: Труды семинара "Симметрии и интегрируемые системы". Ред. А.Н. Сисакян. Дубна: ОИЯИ, 1999. С. 103.

[16] В. Г. Кадьиевский, В. Н. Родионов. ТМФ. 2000. Т. 125. № 3. С. 432.

[17] G. E. Stedman et al. Phys. Rev. A. 1995. V. 51. P. 4944.

[18] В. И. Денисов, И. П. Денисова. ТМФ. 2001. Т. 129. С. 131; Докл. РАН. 2001. Т. 378. C. 4 .

[19] И. М. Тернов, В.Г. Багров, В. Ч. Жуковский. Вестн. МГУ. Сер. физика, астрономия. 1966. № 1. C. 30.

[20] K. Hurley et al. Nature. 1999. V. 397. P. 41; C. Kouveliotou, S. Dieters, T. Strohmayer et al. Nature. 1998. V. 393. P. 235.

[21] R. C. Duncan. Physics in ultra-strong magnetic fields. astro-ph/0002442.

[22] И. М. Тернов, В.Н. Родионов, А.И. Студеникин. Вклад нейтральных слабых токов в аномальный магнитный момент электрона во внешнем поле. В сб.: Тезисы докладов Всесоюзного совещания по квантовой метрологии и фундаментальным физическим константам. Ред. Ю.В. Тарбеев. Л.: НПО "ВНИИМ” им. Д.И. Менделеева, 1982. С. 47; ЯФ. 1983. T. 37. C. 1270 .

[23] В.А. Обухов, В.К. Перес-Фернандес, В. Н. Родионов, В. Р. Халилов. Изв. вузов. Физика. 1983. № 1. С. 26 .

[24] A. Czarnecki, W. Marciano. Phys. Rev. D. 2001. V. 64. P. 012014.

[25] M. Davier, A. Hocker. Phys. Lett. B. 1998. V. 435. P. 427.

[26] M. Davier, S. Eidelman, A. Hocker, Z. Zhang. Eur. Phys. J. C. 2003. V. 27. P. 497; hep-ph/0208177.

[27] B. Krause. Phys. Lett. B. 1997. V. 390. P. 392.

[28] E. Bartoš, S. Dubnička, A.Z. Dubničová, E. A. Kuraev, E. Zemlyanaya. Nucl. Phys. B. 2002. V. 632. P. 330.

[29] E. Bartoš, S. Dubnička, A.Z. Dubničová, E. A. Kuraev, E. Zemlyanaya. Is there any room for new physics in the muon $g-2$ problem? hep-ph/0305051.

[30] C.S. Ozben, G. W. Bennett et al. Muon $g-2$ Collaboration. Precision measurement of the anomalous magnetic moment of the muon. hep-ex/0211044.

[31] G. W. Bennett et al. Muon g-2 Collaboration. Phys. Rev. Lett. 2002. V. 89. P. 1001804.

[32] Л. Б. Окунь. Лептоны и кварки. М.: Наука, 1981.

[33] В. В. Скалозуб. ЭЧАЯ. 1985. Т. 16. С. 1005.

[34] Wu-Yang Tsai, A. Yildiz. Phys. Rev. D. 1971. V. 4. P. 3643.

[35] В. Н. Родионов, Г. А. Кравцова, А. М. Мандель. Письма в ЖЭЭТ. 2002. Т. 75. С. 435; ДАН. 2002. Т. 386. С. 753. 